\title{
A System for Simulating Fluctuation Diagnostics for Application to Turbulence Computations
}

R. V. Bravenec, W. M. Nevins

February 28, 2006

Review of Scientific Instruments 
This document was prepared as an account of work sponsored by an agency of the United States Government. Neither the United States Government nor the University of California nor any of their employees, makes any warranty, express or implied, or assumes any legal liability or responsibility for the accuracy, completeness, or usefulness of any information, apparatus, product, or process disclosed, or represents that its use would not infringe privately owned rights. Reference herein to any specific commercial product, process, or service by trade name, trademark, manufacturer, or otherwise, does not necessarily constitute or imply its endorsement, recommendation, or favoring by the United States Government or the University of California. The views and opinions of authors expressed herein do not necessarily state or reflect those of the United States Government or the University of California, and shall not be used for advertising or product endorsement purposes. 


\title{
A System for Simulating Fluctuation Diagnostics for Application to Turbulence Computations
}

\author{
R. V. Bravenec \\ The University of Texas \\ Fusion Research Center \\ 1 University Station, C1510 \\ Austin, TX 78712-0263 \\ W. M. Nevins \\ Lawrence Livermore National Laboratory \\ P.O. Box 808 (L-637) \\ Livermore, CA 94551-0808
}

Present-day nonlinear microstability codes are able to compute the saturated fluctuations of a turbulent fluid versus space and time, whether the fluid be liquid, gas, or plasma. They are therefore able to determine turbulence-induced fluid (or particle) and energy fluxes. These codes, however, must be tested against experimental data, not only with respect to transport, but also characteristics of the fluctuations. The latter is challenging because of limitations in the diagnostics (e.g., finite spatial resolution) and the fact that the diagnostics typically do not measure exactly the quantities the codes compute. In this work, we present a system based on $\operatorname{IDL}^{\circledR}$ analysis and visualization software in which user-supplied "diagnostic filters" are applied to the code outputs to generate simulated diagnostic signals. The same analysis techniques as applied to the measurements, e.g., digital time-series analysis, may then be applied to the synthesized signals. Their statistical properties, such as rms fluctuation level, mean wave numbers, phase and group velocities, correlation lengths and times, and in some cases full $S(\boldsymbol{k}, \omega)$ spectra can then be compared directly to those of the measurements. 
PACS codes: $02.70 .-\mathrm{c}$, 07.05.Tp, 47.11.+j, 52.65.Tt

\section{Introduction}

A "grand challenge" of magnetic confinement fusion research is to be able to predict the transport in next-step magnetic fusion devices from first-principles theory. Plasma microstability theory has advanced to the point of being able to solve the nonlinear gyrokinetic / Maxwell equations including trapped particles, plasma shaping, multiple species, profile variation within the simulation domain, etc. Because the equations are extremely complex, they can be solved only on massively parallel supercomputers. Different solution schemes - Lagrangian or Eulerian, flux-tube and global - have been employed, and most have and are being benchmarked against each other (e.g., the "Numerical Tokamak"1 and "Cyclone"2 projects) by comparing predictions of thermal diffusivities for a given set of input plasma profiles.

The ultimate benchmark, however, is real-world experimental data, and not only thermal and particle diffusivities (fluxes), but also turbulence parameters (fluctuation amplitude, wavenumber spectra, etc.). Unless the theory (as contained in the numerical algorithms and hereafter referred to as the "code") can predict all these features, the theory is incomplete at best. Comparisons of code results for the turbulence with measurements are difficult because of limitations in the diagnostics (e.g., finite spatial resolution) and the fact that the diagnostics typically do not measure exactly the quantities that the codes compute. For example, in the realm of plasma diagnostics, beam-emission spectroscopy (BES) ${ }^{3}$ measures emission from a beam of neutral atoms, Langmuir probes measure ion saturation current and floating potential, electron-cyclotronemission radiometry measures a mix of electron temperature and density depending on the opti- 
cal thickness, etc. The traditional approach has been to infer local densities, temperatures, etc. (parameters the code calculates) from the measurements, ${ }^{3,4,5,6,7}$ or to model ${ }^{8,9,10,11,12,13}$ the diagnostic, which usually requires assumptions about the nature of the turbulence. The alternative, presented here, is to apply the diagnostic's sensitivities (parameter detected and spatial resolution) to the code output and to generate simulated signals, i.e., a "virtual diagnostic." The statistical properties of the simulated signals can be compared directly with those from the diagnostic, say through time-series analysis.

Virtual diagnostics are valuable not only to help benchmark codes against experimental data, but also for interpretation of turbulence measurements, design of experiments (i.e., if the available diagnostics will be able to resolve the fluctuations predicted by numerical computation in a proposed experiment) and design of diagnostics (i.e., if the proposed diagnostic will be able to resolve the fluctuations predicted by numerical computation in an existing experiment).

A virtual diagnostic for an initial-value turbulence computation is composed of four components: i) an anti-aliasing filter, ii) calculation of the measured parameter (line or black-body emission, phase shift of transmitted or reflected radiation, fluid velocity, etc.) from the code outputs, iii) the effects of finite spatial resolution, and iv) the effects of noise. We address these individually, with examples, in the following sections.

\section{Anti-aliasing}

Sampling a continuous function at a frequency $f_{s}$ can generate spurious features in the frequency spectra due to a phenomena known as aliasing. ${ }^{14}$ An oscillation at frequency $f$ greater than the Nyquist frequency $f_{N}=f_{s} / 2$ will appear in the frequency spectrum at $f_{s}-f$. Therefore, the signal 
being sampled must be low-pass filtered with a cutoff frequency $f_{N}$. Initial-value microstability codes solve their equations by stepping along in discrete time steps. In order for the solutions to converge, the time steps must be much less than the period of any relevant mode in the system. Consequently, aliasing due to the discrete time steps is not a concern. However, because the time step is so small, the output is typically sampled at a subset of time steps for practical reasons (sizes of arrays and files). Then, one must be concerned about aliasing, so like a fluctuation diagnostic, an anti-aliasing filter should be applied to the raw code output before any spectral analysis is performed.

Here we consider a Butterworth filter, a common type of passive filter. The transfer function is

$$
|H(f)|^{2}=\frac{1}{1+\left(f / f_{N}\right)^{2 n}}
$$

where $n$ is the order of the filter. This expression is in terms of frequency whereas we want to apply the filter function to the time-dependent code output. It is well known from Fourier theory that the output of a system is the input convolved with the impulse response of the system given by $^{15}$

$$
h(t)=\int_{-\infty}^{\infty} H(f) e^{i 2 \pi f t} d f
$$

After some complex algebra, this yields (for $n=5$ )

$$
\begin{aligned}
\frac{h(t)}{2 \pi f_{N}}= & \frac{2}{\sqrt{5}} \cos \left(\sqrt{\frac{5+\sqrt{5}}{2}} \pi f_{N} t+\frac{3 \pi}{5}\right) \exp \left(-\frac{\sqrt{5}-1}{2} \pi f_{N} t\right) \\
& -2 \sqrt{1+\frac{2}{\sqrt{5}}} \cos \left(\sqrt{\frac{5-\sqrt{5}}{2}} \pi f_{N} t+\frac{3 \pi}{10}\right) \exp \left(-\frac{\sqrt{5}+1}{2} \pi f_{N} t\right) \\
& +\left(1+\frac{2}{\sqrt{5}}\right) \exp \left(-2 \pi f_{N} t\right)
\end{aligned}
$$


for $t \geq 0$ and zero otherwise (causality). The normalization of $h(t)$ is such that its integral from zero to infinity is unity. The application of such a filter to the raw code output $x(t)$ produces the output

$$
y(t)=\int_{-\infty}^{t} x\left(t^{\prime}\right) h\left(t-t^{\prime}\right) d t^{\prime}
$$

An example of $h\left(t-t^{\prime}\right)$, where $t$ is in units of the code's time step and the sample interval $\Delta t=20$, is shown in Fig. 1. (Here we have plotted $h$ versus $t^{\prime}-t$ to emphasize that the function is applied to previous time steps.) From the figure, we see that application of the anti-aliasing filter requires that the raw code output must be retained for at least the previous 100 (in general, $5 \Delta t$ ) time steps for each sample time. Either the code is required to output its results every time step, after which the anti-aliasing filter is applied to reduce the consequently large file, or the antialiasing filter is incorporated into the code itself, outputting only at the sampling times. Which choice is better depends on the code.

The next three steps of creating a virtual diagnostic depend on the specific diagnostic. It is much easier to describe the steps using an example. We choose here beam-emission spectroscopy (BES), ${ }^{6}$ a plasma fluctuation diagnostic.

\section{Measured Parameter}

BES measures Balmer- $\alpha$ emission from a neutral hydrogen or deuterium beam. The beam is viewed off-perpendicular to provide a Doppler shift to the beam emission. A bandpass filter then filters out emission on either side of the beam lines, specifically the bright unshifted $\mathrm{D}_{\alpha}$ line from the plasma edge. The beam emissivity is given by $n_{b} n_{e} k$, where $n_{b}$ and $n_{e}$ are the beam and electron densities, respectively, and $k$ is the emission coefficient which is a function of beam en- 
ergy, electron and ion densities, impurity charge, and (less so) electron and ion temperatures. ${ }^{6,16,17}$ The emissivity rate $\varepsilon=n_{e} k$ as a function of electron density $n_{e}$, beam energy, and the effective ion charge $Z_{\text {eff }}$ is shown in Fig. 2. The latter is defined through the relation $n_{e} Z_{e f f}=\sum_{j} n_{j} Z_{j}^{2}$, where $n_{j}$ is the density of the $j$ th ion species, and $Z_{j}$ is its charge. Here we consider only one impurity, of density $n_{z}$ and charge $Z$, such that $Z_{\text {eff }}=Z\left(1-n_{i} / n_{e}\right)+1$, where $n_{i}$ is the background ion density. The rates are presented at three beam energies in the ratios $1: 1 / 2: 1 / 3$ not by accident; all neutral beams produce components at these fractional energies due to incomplete dissociation and ionization of molecular hydrogen (or deuterium) in the source. The fraction of the beam density at each energy is specific to the individual beam and must be measured. Note that the rate rolls over at high $n_{e}$ and decreases as $Z_{\text {eff }}$ increases. Therefore, the relative emissivity fluctuations will not follow exactly the electron density fluctuations.

\section{Spatial Resolution}

As an example of a spatial sensitivity function we consider the BES system ${ }^{18}$ on the Alcator CMod tokamak. ${ }^{19}$ The optical system images the beam at a near-vertical plane through the beam axis upon four 1-mm optical fibers in a square pattern. The collection area at the plane is an image of the fiber tips magnified by $\sim 3.5$. Light is collected, however, all along the view chord passing through the near-Gaussian beam profile of e-folding width $\sim 10 \mathrm{~cm}$. The turbulent structures are extended along the magnetic field lines, which pass through the beam perpendicular to its axis. The BES views, however, are not along the field lines. Rather than do the full 3-D problem, we project the BES collection volume onto the vertical plane through the beam axis and approximate the spatial sensitivity function in the form 


$$
f(R, z)=f_{0} \exp \left[-\left(\frac{R-R_{0}}{\delta R}\right)^{\alpha_{R}}\right] \exp \left[-\left(\frac{z-z_{0}}{\delta z}\right)^{\alpha_{z}}\right]
$$

where $R$ and $z$ are the radial and vertical coordinates, respectively ( $R=z=0$ is on the major axis at the midplane of the tokamak), $\left(R_{0}, z_{0}\right)$ are the coordinates of the center of the projected collection volume, $\delta R$ and $\delta z$ are the e-folding radii, and $\alpha_{R}$ and $\alpha_{z}$ are parameters used to control the sharpness of the edges. $f_{0}$ is chosen such that the integral of Eq. (5) over all space is unity. An approximation to the BES sensitivity function at $R_{0}=0.871 \mathrm{~m}, z_{0}=0$ is shown in Fig. 3, where $\delta R=0.8 \mathrm{~cm}, \delta z=0.5 \mathrm{~cm}, \alpha_{R}=4$, and $\alpha_{z}=6$.

There is also a more subtle component of spatial averaging first pointed out in Ref. 6, which we will call beam-emission "smearing." Because of the high directed velocity and finite decay time of an excited beam neutral, the emission will occur "downstream" of the excitation location (where the plasma density information is imprinted on the beam). The emission does not take place at a fixed time after excitation but in a statistical distribution with a characteristic decay time and, because of the beam velocity, length. Therefore, the emission at a viewing point is effectively a weighted average of the emission a decay length "upstream." A rigorous treatment of this effect would require solving the complete set of $1-\mathrm{D}$ (beam direction) rate equations ${ }^{20}$ for every point within the spatial sensitivity function and at every time sample using the 3-D density distributions output by the code. This is not done here because of the high densities and consequently short estimated smearing lengths $(3-4 \mathrm{~mm})^{21}$ for the plasma used in our example. However, in general, smearing could be included within the framework of the system described here. 


\section{Noise}

All diagnostics suffer from noise of some sort. Here we define noise very generally as any component of the signal that is not desired. The specific types of noise are particular to the diagnostic, but all noise is either random or correlated. By random we mean that the noise on a signal is statistically uncorrelated with that signal. For a multi-channel instrument, random noise on the signal from one channel is also uncorrelated with the signal and its noise from a different channel. We do not consider external non-random noise like 50- or 60-Hz pickup (and harmonics) which are relatively easy to account for, if not eliminate.

\section{Random noise}

Random noise is from "external" sources, such as photon noise and thermal noise of electroncyclotron emission $(\mathrm{ECE})^{22}$ and "intrinsic" electronics noise. ${ }^{23}$ The latter include thermal ("Johnson") noise, due to random, thermally induced motion of discrete charge carriers through conductors (including semiconductors) at finite temperature. The mean-squared noise in the current is proportional to the product of temperature and conductance. "Shot" noise is due to the random motion of discrete charge carriers flowing across a potential barrier such as the depletion region of a photodiode. The mean-squared noise in the current is proportional to the average current. The frequency spectra of these sources are flat ("white" noise). Noise sources whose spectra are not flat also exist, but they are typically not important. However, if the detector circuitry contains a number of noise sources and reactive elements, which is almost surely the case because of parasitic capacitances, the noise on the output signal can be substantial and dependent on frequency. In the case of the BES systems on the Alcator C-Mod and DIII-D tokamaks, the electronics noise increases monotonically with frequency ${ }^{24}$ up to the cutoff frequency of the antialias filters. 
Random noise is relatively simple to extract, statistically, from the total fluctuation spectrum because it is uncorrelated with the desired signal, by definition. One first measures the electronics noise autopower spectrum $N(f)$ by turning off the source of the desired signal, shielding the detector, withdrawing the probe, etc. (Here, $N(f) \equiv\left\langle n_{f} n_{f}^{*}\right\rangle$, where $n_{f}$ is the Fourier transform of the noise signal $n(t), n_{f}^{*}$ is its complex conjugate, and the angled brackets denote an ensemble average.) One then simply subtracts $N(f)$ from the autopower spectrum of the data. The remainder is the spectrum of the desired signal plus external noise. Since photon or ECE thermal noise is white, a baseline may be imposed on the difference spectrum within a frequency range where the desired signal is known to vanish, e.g., at high frequencies. If such a frequency range is not evident, or there is little remaining after subtracting the intrinsic noise, then cross-correlation between signals from different detectors is necessary, e.g., the crosspower $\left\langle s_{1 f} s_{2 f^{*}}\right\rangle$, where $s_{i}$ is the signal from detector $i$ and the other notation is as described above. This effectively eliminates all random noise except for a small residue because of a finite number of samples (which itself can be eliminated by a slight modification to the standard crosspower ${ }^{3}$ ). Of course, if the two detectors are viewing different locations, spatial decorrelation between the signals must be taken into account.

Random noise sources may be modeled in the computations, but this would be much more work than accounting for the noise in the measurements, as described above. We should point out that numerical (statistical) noise in the computations may also be a concern. Lagrangian (moving grid) particle-in-cell (PIC) codes suffer from noise related to sampling error which accumulates during a run. ${ }^{25}$ If incorporating virtual diagnostics into such a code, the user must be aware of 
and properly account for this noise. In contrast, codes utilizing an Eulerian algorithm (fixed grids) are intrinsically free of such noise. In either case, however, the computations suffer from short duration compared to measurements since the computations are very expensive with regard to computer resources. However, they enjoy high spatial resolution. Therefore, the statistics can be improved by averaging over ensembles made up of time series from different locations with the same statistical properties but uncorrelated, e.g., points sufficiently spaced along an ignorable coordinate. This can be done instead of, or in addition to, averaging over sub-samples of a single time history. In this way, the total number of "data" points, and therefore the statistical uncertainties, can be made the same as the measurement.

\section{Correlated Noise}

Noise which is either correlated with the desired signal or between channels is much harder to extract from the raw data. Such noise is best illustrated by example, in particular BES, which can suffer from many sources of correlated noise. BES detects all emission within a defined wavelength band along view chords through the plasma. In addition to Balmer- $\alpha$ emission from

the beam, BES also detects bremsstrahlung (broadband and proportional to $n_{e}{ }^{2} Z_{e f f}$ ) from all points in the plasma within the acceptance of the optics, impurity line emission from radii dependent on the specific line(s) and that falls within the filter passband, and edge $\mathrm{D}_{\alpha}$ that "leaks" into the filter passband. This "background" emission is undesirable because it is mostly nonlocal. This is ameliorated by the natural defocusing of a typical optical system away from the focus (the measurement volume). The rate of defocusing is proportional to the inverse of the input $f$-number of the optics. The larger collection volume away from the focus act to average out the small-scale fluctuations. Nevertheless, the quantitative extent to which this localizes the measurement is not known a priori. Even if some of the emission originates from the measurement 
volume, the fluctuations are different functions of plasma parameters than the beam emission, so in combination it is not clear what fluctuations are being measured. At the least, the background fluctuations interfere with quantitative measurements, e.g., electron density fluctuation level.

If this background emission does not change when the beam is turned on (a reasonable assumption for a diagnostic neutral beam, and one that can be verified), one may measure the spectrum before and/or after the beam pulse. If the background fluctuations were uncorrelated with the beam emission, their autopower spectrum could be subtracted from that measured during the beam - similar to the way random noise can be extracted from the signals. This would be valid for removing broadband edge $\mathrm{D}_{\alpha}$ fluctuations since they originate outside the last closed flux surface and therefore would be uncorrelated with interior fluctuations. (This may not be the case for large-scale intermittent or semi-coherent structures such as ELM's, ${ }^{26}$ avalanches,${ }^{27}$ quasicoherent modes, ${ }^{28}$ edge harmonic oscillations ${ }^{29}$ etc., which could manifest themselves throughout the plasma. In the case of relatively infrequent intermittent structures, like some ELM's, one could simply isolate the structures temporally and eliminate them from the data set. ${ }^{30}$ ) The same applies to impurity line emission if it originates well outside the measurement volume. However, there are always bremsstrahlung fluctuations from the measurement volume which are therefore correlated with the beam-emission fluctuations. Since bremsstrahlung is proportional to $n_{e}^{2}$, BES would be more than twice as sensitive to these fluctuations as to those from the beam. In general then, one cannot simply subtract the background spectrum from the beam spectrum unless the signal during the beam is much larger than before or after. In contrast to random noise, correlation analysis of signals from nearby channels does not help. This is not only because each noise source is correlated between channels, but also because the phase of the 
crosspower (or time shift of the correlation function) of the beam emission cannot be extracted from the measured phase (or time shift).

If the bremsstrahlung emission is of order the beam emission, modeling of the former in a virtual BES diagnostic is called for. Emission along the entire view chord should be calculated from the computation (the spatial sensitivity function discussed earlier should be fully 3-D), requiring a global computation from the measurement volume out to the edge. (If the contribution of the edge region is subsequently found to contribute little to the simulated signals, a flux-tube computation would then be sufficient.) If significant impurity line emission exists within the filter passband and from the measurement volume, it would need to be modeled as well. The computation should include the dynamics of the impurity species observed in addition to the dominant species determining $Z_{\text {eff }}$, if different. The virtual BES, in turn, must model the dependences of the emission on impurity and plasma density, temperatures, etc. If correlation analysis is intended, then impurity line emission must be modeled even if it originates from outside the measurement volume. In principle, this should also apply to edge $\mathrm{D}_{\alpha}$ emission, if significant, although the codes presently available are not valid in the very edge where the $\mathrm{D}_{\alpha}$ emission originates. Therefore, correlation analysis would be precluded in this scenario.

A similar yet even more problematic correlated noise source is modulation of the beam density at the observation point by fluctuations in the plasma density upstream. This occurs through modulation of the cross sections for ionization and/or charge-exchange loss of beam particles - the beam density at the observation point drops when the plasma density upstream increases, and vice-versa. Just as for beam smearing discussed earlier, a rigorous treatment of this effect would 
require solving the complete set of rate equations (including ionization and charge exchange) for every point within the observation volume and at every time sample using the 3-D density distributions output by the code. As in the case of random noise, it is more sensible to account for this effect (for broadband turbulence) in the measurements themselves, as demonstrated in Ref. 31. Like edge $\mathrm{D}_{\alpha}$ contamination, this effect could not be modeled well if the density fluctuations peak outside the radial range of validity of the code.

None of the modeling of correlated noise is performed here, mostly because it is beyond the scope of this work. We would simply stress that modeling of background emission (other than perhaps edge $D_{\alpha}$ ), beam smearing, and beam attenuation modulation is possible, albeit difficult, within the framework described here.

\section{Analysis}

Application of a virtual diagnostic to the code outputs is performed within an interactive data analysis and visualization tool called GKV ("GyroKinetic Visualization") ${ }^{32}$ which is specifically designed for analyzing output from initial-value microstability codes. It is an object-oriented ${ }^{33}$ application built upon IDL ${ }^{\circledR}$ (Interactive Data Language). ${ }^{34}$ The code outputs, e.g., electron, ion, and impurity densities, stored as functions of time in netCDF ${ }^{35}$ or text files, are imported into GKV by procedures specific to the particular microstability code and then transformed into IDL ${ }^{\circledR}$ objects. The objects can have as many as three spatial dimensions plus time. Analysis and visualization are performed within GKV through "methods" which act on the objects to produce new objects. This system enhances the power of the native IDL ${ }^{\circledR}$ language, making it possible to perform significant amounts of data analysis and to produce presentation-quality plots with relatively few lines of IDL script. 
The virtual diagnostics are coded as methods within this framework. For our BES example, calculation of emissivity from the plasma parameters is coded in the method BES_Emiss which is called as follows:

emiss_rate $=n e \_i n->B E S \_E m i s s$ (ni=ni_in, $Z=Z \_i n, E b=E b \_$in, fracts $=$fracts_in).

The inputs $n e$ in and $n i$ in are 3-D objects, functions of $R, z$, and $t$, containing the electron and main ion densities obtained from the code output files. The other keywords are scalars: $Z$ is the (single) impurity charge (not $Z_{\text {eff }}$ ), Eb is the full energy of the beam, and fracts is a three-element array containing the detected fractions of full-, half-, and third-energy beam components. (Because of the different Doppler shifts of each component and a finite filter passband, these fractions may not correspond to the density fractions at the observation point.) The result emiss_rate is an object of the same dimensionality as ne in containing the emissivity rate.

Similarly, the spatial averaging is performed by a method Project, which is called as follows:

signal $=$ emiss_rate $->$ Project $\left(\right.$ Axis $=$ axis, Center $=\left[R_{0}, z_{0}\right]$, Width $=[\delta R, \delta z]$, Power $\left.=\left[\alpha_{R}, \alpha_{z}\right]\right)$.

Project multiplies emiss_rate by the 2-D weighting function given in Eq. (5) earlier $\left(f_{0}\right.$ is computed internally), integrates over the extent of the domain, and returns the object signal (a function of time only). The input keyword Axis is the numerical identifier of the dimension not being averaged over (time). The other keywords were defined earlier in the context of Eq. (5). The surface plot in Fig. 3 was generated by the subsidiary method ProjectWeight via the command 
weight $=$ emiss_rate- $>$ ProjectWeight $\left(\right.$ Center $=\left[R_{0}, z_{0}\right]$, Width $=[\delta R, \delta z]$, Power $\left.=\left[\alpha_{R}, \alpha_{z}\right]\right)$.

Before leaving this section, we should point out that Project could be generalized by having as inputs an arbitrary 2- or 3-D array of weights on an accompanying 2- or 3-D grid rather than an analytic function such as Eq. (5). If 3-D, however, the code outputs would have to be available and read into GKV in all three spatial dimensions.

\section{Example}

We now apply the above virtual diagnostic methods to a turbulence computation for an enhanced $\mathrm{D}_{\alpha} \mathrm{H}$-mode discharge ${ }^{36}$ on the Alcator C-Mod tokamak in the vicinity of $R=R_{0}=0.871 \mathrm{~m}, z=$ $z_{0}=0$. The motivation for choosing this discharge and location is not to benchmark the computation against fluctuation data; in fact, the BES diagnostic detects no broadband fluctuations here, even though estimates of the signal-to-noise ratio indicate it should. Since there are no other fluctuation diagnostics in this region to help explain this, we turn to "virtual BES" for insight.

We employ the GYRO code, ${ }^{37}$ which solves the nonlinear gyrokinetic / Maxwell equations on a fixed spatial grid in a standard toroidal coordinate system $(r, \theta, \phi)$ with $r$ the minor radius accounting for plasma shaping ( $r=0$ corresponds to the magnetic axis) , $\theta$ the "poloidal" angle between $\boldsymbol{r}$ and the outer midplane, and $\phi$ the toroidal angle. The computation domain is an annular wedge centered at $\left(R_{0}, z_{0}\right)$, corresponding to $r=r_{0}=0.192 \mathrm{~m}, \theta=0$, and extended along the

field lines. It is of dimensions $100 \rho_{s}$ (radial) by $230 \rho_{s}$ (poloidal) ( $\rho_{s}$ is the ion gyroradius at 
the electron temperature). We assume periodic radial boundary conditions and utilize 32 toroidal modes and 128 radial grid points. Because of the low plasma pressure in the periphery, we assume the turbulence is purely electrostatic. However, we include kinetic electrons and one impurity species (boron). The IDL ${ }^{\circledR}$ code GS2_PREP ${ }^{38}$ is used to calculate input parameters to GYRO from TRANSP ${ }^{39}$ analysis of the discharge.

GYRO is run until a saturated turbulent state is obtained, which is typically at least $200 a / c_{s}$, where $a$ is the plasma minor radius $(0.221 \mathrm{~m})$ and $c_{S}$ is the ion sound speed $\left(1.63 \times 10^{5} \mathrm{~m} / \mathrm{s}\right)$, and then as long as possible afterwards to provide adequate statistics. The computation presented here was run on the IBM SP RS/6000 machine "Seaborg" at the National Energy Research Scientific Computing Center (NERSC) out to $\sim 800 a / c_{s}$, consuming $\sim 20,000$ processor-hours. Data was output every unit of $a / c_{s}$, resulting in $\sim 600$ sample times for analysis. (These, of course, are much fewer samples than a typical measurement. More will be said about this in a later section. Even so, the output was considerably over-sampled and the anti-aliasing filter had no effect.)

We should point out that our use of radially periodic boundary conditions in a flux-tube computation domain means that the computation is performed in a rotating frame where there is no background electric field $\mathbf{E}$. Measurements, however, are made in the laboratory frame, where in general $\mathbf{E} \neq \mathbf{0}$. The electric field acts to shift the measured frequencies of the fluctuations by the amount $\Delta \omega=\mathbf{k} \cdot \mathbf{v}_{\mathbf{E}} \mathbf{B}$. Here $\mathbf{k}$ is the wave vector and $\mathbf{v}_{\mathbf{E}_{\mathbf{\times}} \mathbf{B}}=\mathbf{E} \times \mathbf{B} / B^{2}$, where $\mathbf{B}$ is the total magnetic field. Since typically $E_{r}>>E_{\phi}\left(E_{\theta} \equiv 0\right)$ and $B_{\phi}>>B_{\theta}\left(B_{r} \equiv 0\right)$, then $v_{\mathbf{E}_{\mathbf{x}} \mathbf{B}} \approx-E_{r} / B_{\phi}$ in the poloidal direction. Therefore, $|\Delta \omega| \approx k_{\theta} E_{r} / B_{\phi}$. In addition, modification of the turbulence (including stabilization) by radial shear in the electric field ${ }^{40}$ is not included in the computation. 
Since $\mathbf{E}$ is not measured in the region of the plasma considered here and we will not be comparing computation results to measurements in detail, these points are moot for the purposes of this work. However, in general, comparisons between simulated and measured spectra must take these effects into account. If instead a global computation were carried out, the background electric field, if measured, could be included. Then care must be taken to sample the code output frequent enough to resolve the typically higher frequencies due to the $\mathbf{E} \times \mathbf{B}$ shift.

A "snapshot" of the electron density distribution in the $R$-z plane is shown in Fig. 4. The poloidal extent represents a full period at $r=r_{0}$ of the lowest-order mode. The main ion density distribution is very similar. Structures are evident that are of the order or smaller than about $1 \mathrm{~cm}$. Although not shown, the impurity density distribution is of even smaller scale. We therefore can expect significant spatial filtering by the weighting function of Fig. 3 .

We first transform the densities into the emissivity rate by applying BES_emiss discussed earlier, with $Z=5$ and the default values of $E b(50 \mathrm{keV})$ and fracts $([0.7,0.2,0.1])$ Because the relative density fluctuations are small, the emissivity-rate distributions look very similar to Fig. 4.

Therefore, we examine the time "signals" at $r=r_{0}, \theta=0$. These are obtained by twice applying the GKV method Slice to the emissivity rate - once to select $r=r_{0}$ and again to select $\theta=0-$ yielding a 1-D function of time. The results for the densities normalized to their time averages are shown in Fig. 5, where we observe that the electron and ion density fluctuations are quite similar, whereas the impurity density fluctuations are of smaller amplitude. We next apply Slice to the emissivity rate, and compare its normalized signal to that of the electron density in Fig. 6. 
We see that there is an attenuation of a factor of about two from electron density to emissivity fluctuations.

Next we investigate the effects of the finite collection volume of the diagnostic. First, we calculate the emissivity rate $\varepsilon$ at $r=r_{0}$ versus $s=r_{0} \theta$ and $t$ by applying Slice to emiss_rate to select $r$ $=r_{0}$. The result is shown in Fig. 7(a). The downward propagation of the fluctuations is clear.

Rather than compute the spatially filtered distribution $\varepsilon_{f}\left(r_{0}, s, t\right)$ by applying Project to emiss_rate multiple times in a poloidal scan (a convolution of the weighting function with the fluctuation distribution), we make use of a well-known Fourier identity and write

$$
\varepsilon_{f}(s, t)=\int_{-\infty}^{\infty} d s^{\prime} \varepsilon\left(s^{\prime}, t\right) f\left(s-s^{\prime}\right)=\Im^{-1}\left\{\varepsilon_{k_{\theta}}\left(k_{\theta}, t\right) f_{k_{\theta}}\left(k_{\theta}\right)\right\}
$$

where $k_{\theta}$ is the poloidal wave number, $\varepsilon_{k_{\theta}}$ and $f_{k_{\theta}}$ are the Fourier transforms of the emissivity rate and weighting function, respectively (in poloidal arc length), and $\Im^{-1}$ is the inverse Fourier transform operator. This identity replaces a convolution integral by a simple product of the Fourier transforms, followed by the inverse Fourier transform. Doing so (actually using the fast Fourier transform (FFT), and inverse FFT) results in the distribution indicated in Fig. 7(b). Compared to (a), not only are the structures blurred, but the amplitude of the fluctuations is reduced as well. This is made more apparent by applying Slice once again, this time to select $\theta=$ 0. The time traces corresponding to Fig. 7(a) and (b) at $\theta=0$ are shown in Fig. 8(a) and (b), respectively. We observe a serious attenuation in the signal $s(t)$, not only in the overall amplitude, but particularly in the higher frequency components. 
These results can be made more quantitative by calculating the frequency spectra. In fact, we can compare only statistical quantities such as spectra with measurements, as there is no possibility (or intent) to generate the exact time series as seen in experiment. As mentioned earlier, the relatively few time samples from the code compared with experiment make conventional timeseries statistical analysis difficult. However, what the code output lacks in time samples, it makes up in spatial samples. Therefore, we use time traces at multiple spatial locations as our ensemble instead of subdivisions of a relatively short time trace. Ideally, the spatial ensemble should be made up of signals at different toroidal angles since toroidal angle is an ignorable coordinate in a tokamak. However, this would require outputting the densities versus all three dimensions and time - an intractable amount of data. Therefore, we use poloidal angle as a proxy for toroidal angle. We can do this because fluctuations are highly correlated along field lines: A fluctuation at a given point is virtually the same at other points on the same field line but toroidally (and consequentially poloidally) displaced.

We therefore consider the autopower spectrum $\left\langle\varepsilon_{\omega} \varepsilon_{\omega}{ }^{*}\right\rangle$ at $r=r_{0}$ averaged over poloidal angle across the simulation domain, where $\omega$ is the angular frequency, $\varepsilon_{\omega}$ is the Fourier transform of $\varepsilon$ and $\varepsilon_{\omega}^{*}$ is its complex conjugate. To do this, we first make use of the GKV method XSpect, which computes the spatial and temporal autopower (or crosspower if a reference object is included in the calling arguments): Emiss_rate $\left(k_{\theta}, \omega\right)=$ Emiss_rate $(s, t)->X S p e c t()$. Because XSpect computes both spatial and temporal spectra, we utilize the identity

$$
\int_{-\infty}^{\infty} d s \varepsilon_{\omega}(s, \omega)=\int_{-\infty}^{\infty} d k_{\theta} \varepsilon_{k_{\theta}, \omega}\left(k_{\theta}, \omega\right)
$$


to average the spectra over poloidal angle, where $\varepsilon_{k_{\theta}, \omega}$ is the Fourier transform in poloidal arc length and time, i.e., the output of XSpect. Thus, integrating over $k_{\theta}$ is equivalent to integrating over space.

The results of applying these operations to the distributions of Fig. 7 is shown in Fig. 9. It is clear that the spatial weighting function of Fig. 3 dramatically attenuates the higher frequencies (corresponding to the larger wave numbers) and even attenuates the lower frequencies. We believe this to be the reason BES detects no turbulence in this discharge.

\section{Conclusions}

We have demonstrated a system based on the GKV data analysis and visualization tool by which "signals" characteristic of a particular diagnostic may be generated from the output of an initialvalue microstability code and then compared with actual diagnostic signals in a statistical sense. Although we have presented only frequency spectra here to show the effects of the diagnostic "filters," higher-order statistical quantities like bispectra, cross-power spectra, cross-bispectra, etc., could be calculated for the simulated signals. These would provide additional information about nonlinear energy exchange processes, generation of zonal flows, and other fundamental properties of the turbulence. Comparisons of these quantities to those from measurements would provide additional evidence one way or the other of the code's validity and would be a worthwhile subject of future work.

Although we use for our example beam-emission spectroscopy applied to a nonlinear gyrokinetic microstability calculation, the system can be applied to virtually any diagnostic and any type of computation, subject only to the capabilities of the user to correctly model the diagnostic. The 
signals generated by the "virtual diagnostic" may be analyzed exactly like those from the actual diagnostic. The primary purpose of doing so is in benchmarking the code, i.e., determining if the code results are consistent with measurements. Once confidence exists that the code faithfully reproduces experiment, then the virtual diagnostic technique may be used to guide the design, implementation, and interpretation of diagnostics and/or experiments.

\section{Acknowledgments}

The authors wish to thank Jeff Candy and Ron Waltz for their assistance with running the GYRO code and Darin Ernst for use of his software GS2_PREP. The first author would like to thank Alan Wootton and Bill Rowan for their motivation of this work, and to acknowledge Dave Ross and Jean-Noel LeBouef for their early work on this topic. Thanks are extended to Ian Hutchinson and Martin Greenwald for helpful discussions about electronics noise and correlated noise, respectively. This work was supported by USDOE under grants DE-FG03-97ER54415 and DE-FG03-96ER-54373, and contract no. W-7405-Eng-48 with UC, LLNL. 


\section{References}

${ }^{1}$ S. E. Parker, W. Dorland, R. A. Santoro, M. A. Beer, Q. P. Liu, W. W. Lee, and G. W. Hammett, Phys. Plasmas 1, 1461 (1994).

${ }^{2}$ A. M. Dimits, G. Bateman, M. A. Beer, B. I. Cohen, W. Dorland, G. W. Hammett, C. Kim, J. E. Kinsey, M. Kotschenreuther, A. H. Kritz, L. L. Lao, J. Mandreakas, W. M. Nevins, S. E. Parker, A. J. Redd, D. E. Schumaker, R. Sydora, and J. Weiland, Phys. Plasmas 7, 969 (2000).

${ }^{3}$ G. R. McKee, R. Ashley, R. Durst, R. Fonck, M. Jakubowski, K. Tritz, K. Burrell, C.

Greenfield, and J. Robinson, Rev. Sci. Instrum. 70, 913 (1999); ibid., 70, 2179(E) (1999).

${ }^{4}$ D. W. Ross, P. M. Schoch, J. W. Heard, T. P. Crowley, and R. L. Hickok, Nucl. Fusion 31, 1355 (1991).

${ }^{5}$ D. W. Ross, M. L. Sloan, A. J. Wootton, P. M. Schoch, J. W. Heard, T. P. Crowley, R. L. Hickok, and V. Simcic, Rev. Sci. Instrum. 63, 2232 (1992).

${ }^{6}$ R. J. Fonck, P. A. Duperrex, and S. F. Paul, Rev. Sci. Instrum. 61, 3487 (1990).

${ }^{7}$ R. V. Bravenec and A. J. Wootton, Rev. Sci. Instrum. 66 (1995) 802.

${ }^{8}$ E. Mazzucato, Rev. Sci. Instrum. 69, 1691 (1998).

${ }^{9}$ B. I. Cohen, E. B. Hooper, T. B. Kaiser, E. A. Williams, and C. W. Domier, Phys. Plasmas 6, 1732 (1999).

${ }^{10}$ Y. Lin, R. Nazikian, J. H. Irby, and E. S. Marmar, Plasma Phys. Control. Fusion 43, L1 (2001).

${ }^{11}$ E. J. Valeo, G. J. Kramer and R. Nazikian, Plasma Phys. Control. Fusion 44, L1 (2002).

${ }^{12}$ G. J. Kramer, R. Nazikian, and E. J. Valeo, Plasma Phys. Control. Fusion 44, L11 (2002).

${ }^{13}$ A. G. Lynn, P. E. Phillips, A. E. Hubbard, and S, J, Wukitch, Plasma Phys. Control. Fusion 46, A61-A70; A. Lynn, Ph. D. thesis, Univ. of Texas at Austin, 2004. 
${ }^{14}$ J. S. Bendat and A. G. Piersol, Random Data: Analysis and Measurement Procedures, $2^{\text {nd }}$ ed. (Wiley-Interscience, New York, 1986), pp. 338-339.

${ }^{15}$ ibid., p. 28.

${ }^{16}$ W. M. Mandl, R. C. Wolf, M. G. von Hellermann, and H. P. Summers, Plasma Phys. Control. Fusion 35, 1373 (1993).

${ }^{17}$ I. H. Hutchinson, Plasma Phys. Control. Fusion 44, 71 (2002).

${ }^{18}$ N. P. Basse, et al., to be published in Fusion Sci. Tech.

${ }^{19}$ I. H. Hutchinson, et al., Phys. Plasmas 1, 1511 (1994).

${ }^{20}$ T. A. Gianakon, R. J. Fonck, J. D. Callen, R. D. Durst, J. S. Kim, and S. F. Paul, Rev. Sci. Instrum. 63, 4931 (1992).

${ }^{21}$ M. B. Sampsell, "Beam Emission Spectroscopy on the Alcator C-Mode Tokamak," Ph.D. dissertation, Univ. of Texas, Austin, Dec., 2004.

${ }^{22}$ G. Cima, Rev. Sci. Instrum. 63, 4630 (1992).

${ }^{23}$ P. J. Fish, Electronic Noise and Low Noise Design, (McGraw Hill, New York, 1994).

${ }^{24}$ R. J. Fonck, R. Ashley, R. Durst, S. F. Paul, and G. Renda, Rev. Sci. Instrum. 63, 4924 (1992).

${ }^{25}$ W. M. Nevins, to be published in Phys. Rev. Lett.

${ }^{26}$ F. Wagner, et al., J. Nucl. Matrl. 121,103 (1984).

${ }^{27}$ P. A. Politzer, M. E. Austin, M. Gilmore, G. R. McKee, T. L. Rhodes, C. X. Yu, E. J. Doyle, T. E. Evans, and R. A. Moyer, Phys. Plasmas 9, 1962 (2002).

${ }^{28}$ M. Greenwald, R. Boivin, P. Bonoli, R. Budny, et al., Phys. Plasmas 6, 1943 (1999).

${ }^{29}$ C. M. Greenfield, K. H. Burrell, J. C. DeBoo, et al., Phys. Rev. Lett. 86, 4544 (2001).

${ }^{30}$ G. R. McKee, private communication, Oct., 2005. 
${ }^{31}$ R. D. Durst, R. J. Fonck, G. Cosby, H. Evensen, and S. F. Paul, Rev. Sci. Instrum. 63, 4907 (1992).

${ }^{32}$ W. M. Nevins, Lawrence Livermore National Laboratory Report UCRL-TR-206016, August, 2004. The GKV project file is available by contacting the author.

${ }^{33}$ A. J. Riel, Object-Oriented Design Heuristics, (Addison -Wesley, New York, 1996).

${ }^{34}$ Research Systems Inc., http://www.rsinc.com/idl/

35 http://my.unidata.ucar.edu/content/software/netcdf/index.html

${ }^{36}$ M. Greenwald, et al., Phys. Plasmas 6, 1943 (1999).

${ }^{37}$ J. Candy, R. E. Waltz, J. Comput. Phys. 186, 545 (2003).

${ }^{38}$ D. R. Ernst, P. T. Bonoli, P. J. Catto, W. Dorland, C. L. Fiore, R. S. Granetz, M. Greenwald, A. E. Hubbard, M. Porkolab, M. H. Redi, J. E. Rice, K. Zhurovich, and the Alcator C-Mod Group, Phys. Plasmas 11, 2637 (2004).

${ }^{39}$ R. J. Goldston, D. C. McCune, H. H. Towner, S. L. Davis, R. J. Hawryluk, and G. L. Schmidt, J. Comput. Phys. 43, 61 (1981).

${ }^{40}$ K. H. Burrell, Phys. Plasmas 4, 1499 (1997) and references therein. 


\section{Figure Captions}

Fig. 1. Unit impulse response of a 5-pole Butterworth anti-aliasing filter for a sampling interval of 20 time steps.

Fig. 2. Emissivity rate of a neutral hydrogen beam as a function of electron density, beam energy, and plasma effective ion charge $Z_{e f f}$, for $k T_{e}=k T_{i}=10 \mathrm{keV}$. (Adapted from Ref. 16.)

Fig. 3. Model of BES sensitivity function at $R=R_{0}=0.871 \mathrm{~m}, z_{0}=z_{0}=0$. The other parameters in Eq. (5) are $\delta R=0.8 \mathrm{~cm}, \delta z=0.5 \mathrm{~cm}, \alpha_{R}=4, \alpha_{z}=6$.

Fig. 4. Snapshot of the electron density distribution (units of $10^{19} \mathrm{~m}^{-3}$ ) at $t=800 a / c_{s}(1.1$ ms) at the outer midplane from the GYRO calculation.

Fig. 5. Time traces of a) electron, b) ion, and c) impurity (boron) densities at $R=0.871 \mathrm{~m}, \boldsymbol{z}$ $=0$ normalized to their time-averages of $20.2,17.2$, and 0.616 (units of $10^{19} \mathrm{~m}^{-3}$ ), respectively.

Fig. 6. Time traces of a) electron density (same as in Fig. 5(a)) and b) emissivity rate at $R=$ $0.871 \mathrm{~m}, z=0$ normalized to their time-averages of $20.2 \times 10^{19} \mathrm{~m}^{-3}$ and $3.21 \times 10^{5} / \mathrm{s}$, respectively.

Fig. 7. Plots of emissivity rate at $\boldsymbol{r}=\mathbf{0 . 1 9 2} \mathrm{m}$ versus poloidal distance from midplane and time (units of $a / c_{s}$ ) for a) perfect spatial resolution and b) applying the spatial sensitivity function of Fig. 3. 
Fig. 8. Time traces of normalized a) emissivity rate at $\boldsymbol{R}=0.871 \mathrm{~m}, z=0$ [same as in Fig. 6(b)] and b) signal after spatial sensitivity function of Fig. 3 is applied.

Fig. 9. Frequency autopower spectra of the time traces in Fig. 8. The light curve corresponds to Fig. 8(a) and the dark curve to Fig. 8(b). 


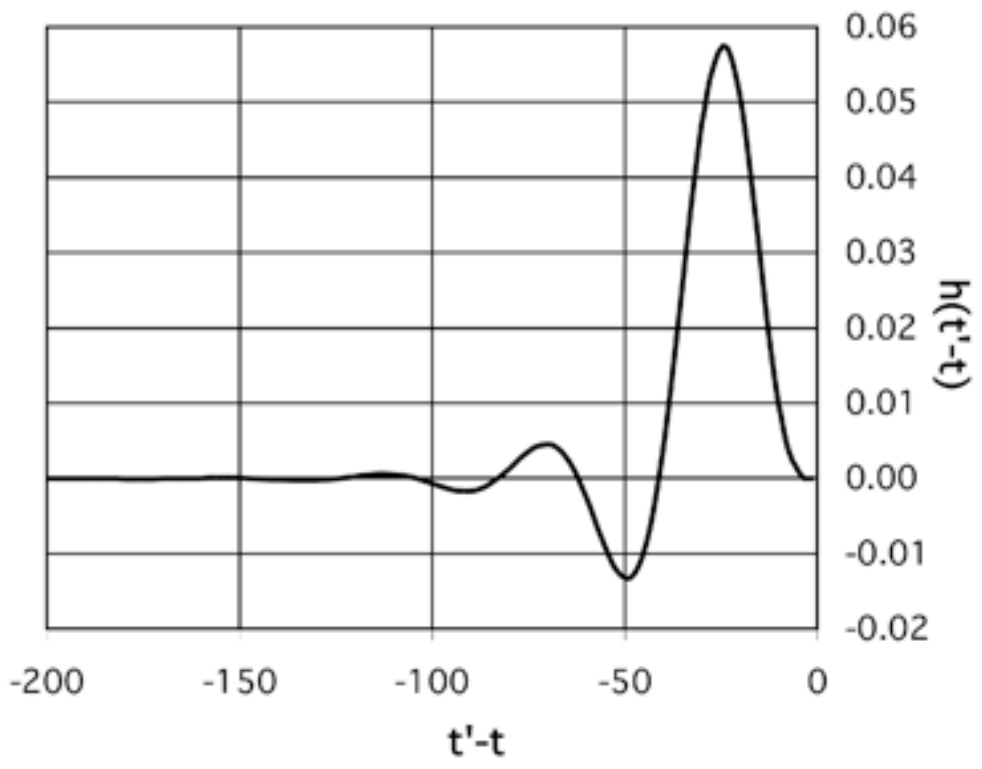

Fig. 1 


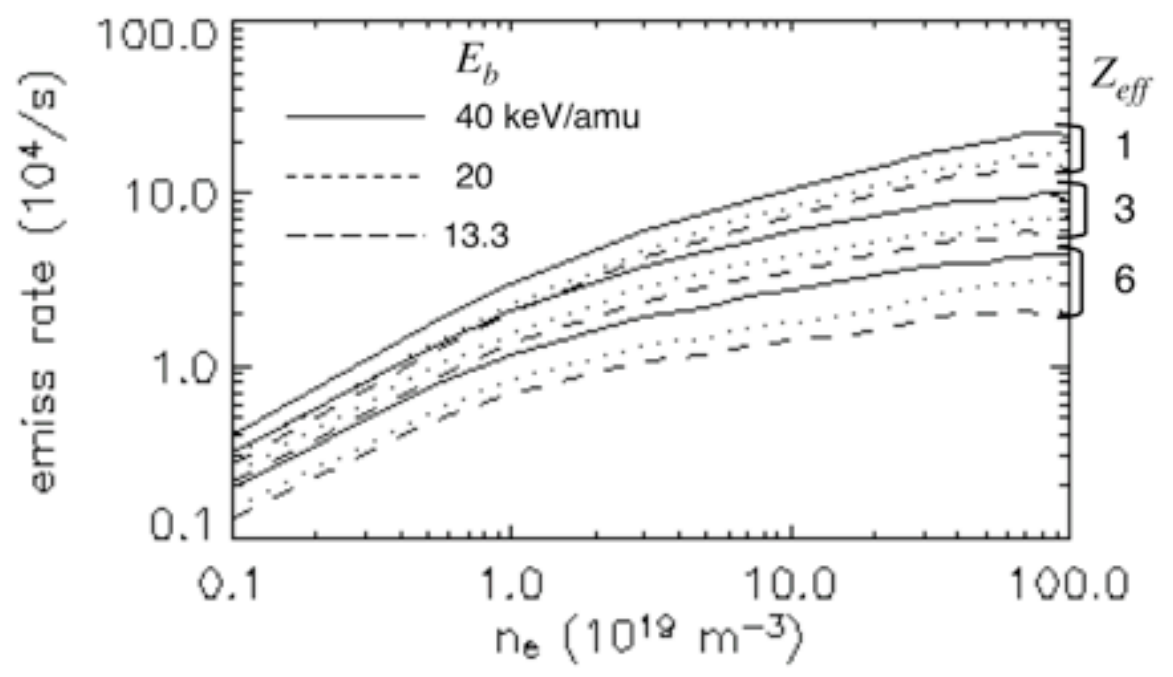

Fig. 2 


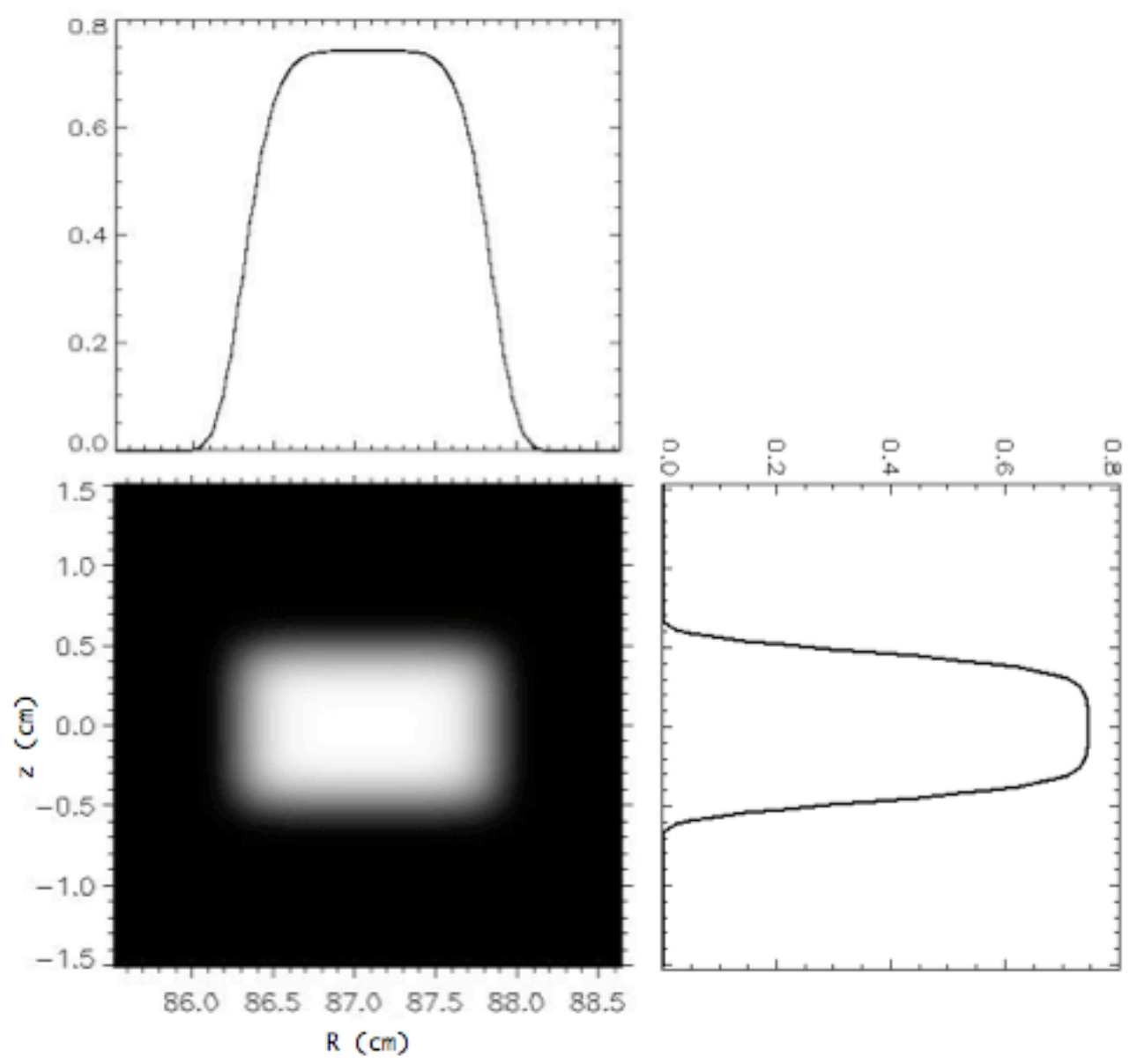

Fig. 3 


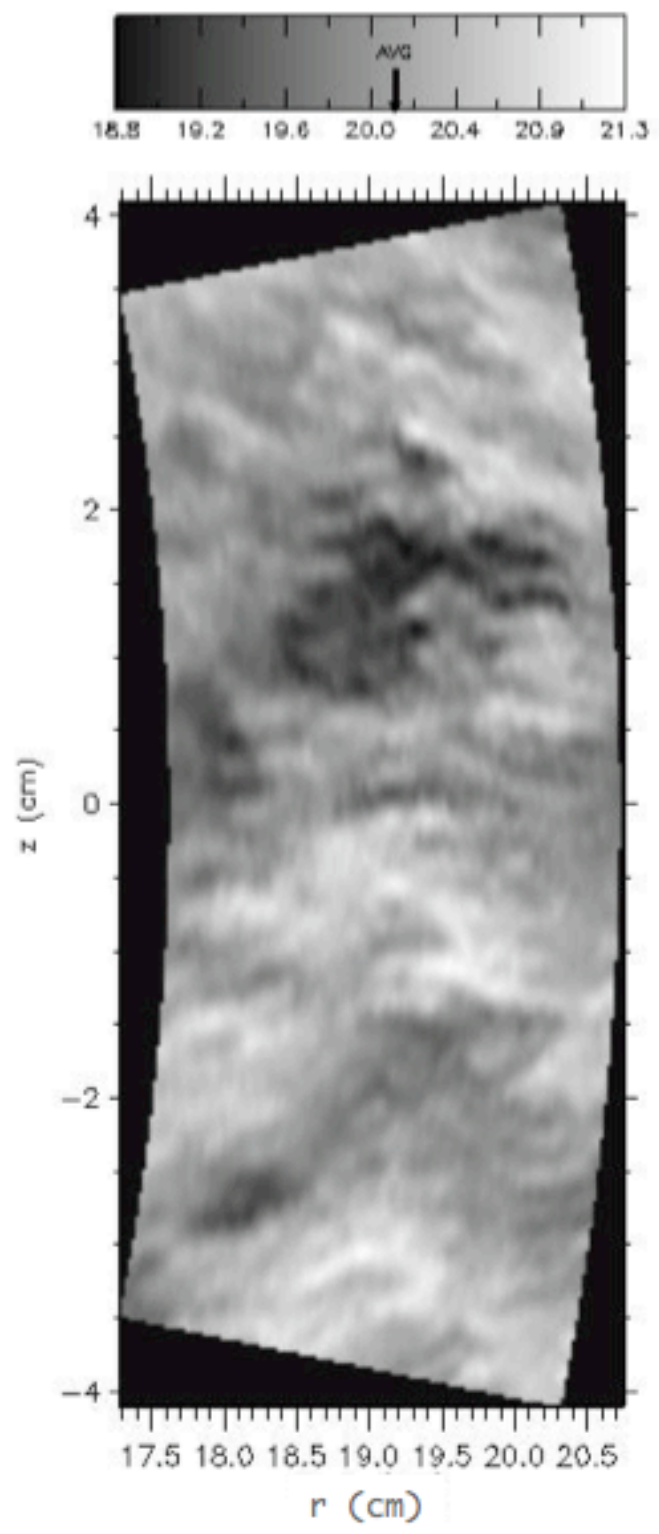

Fig. 4 

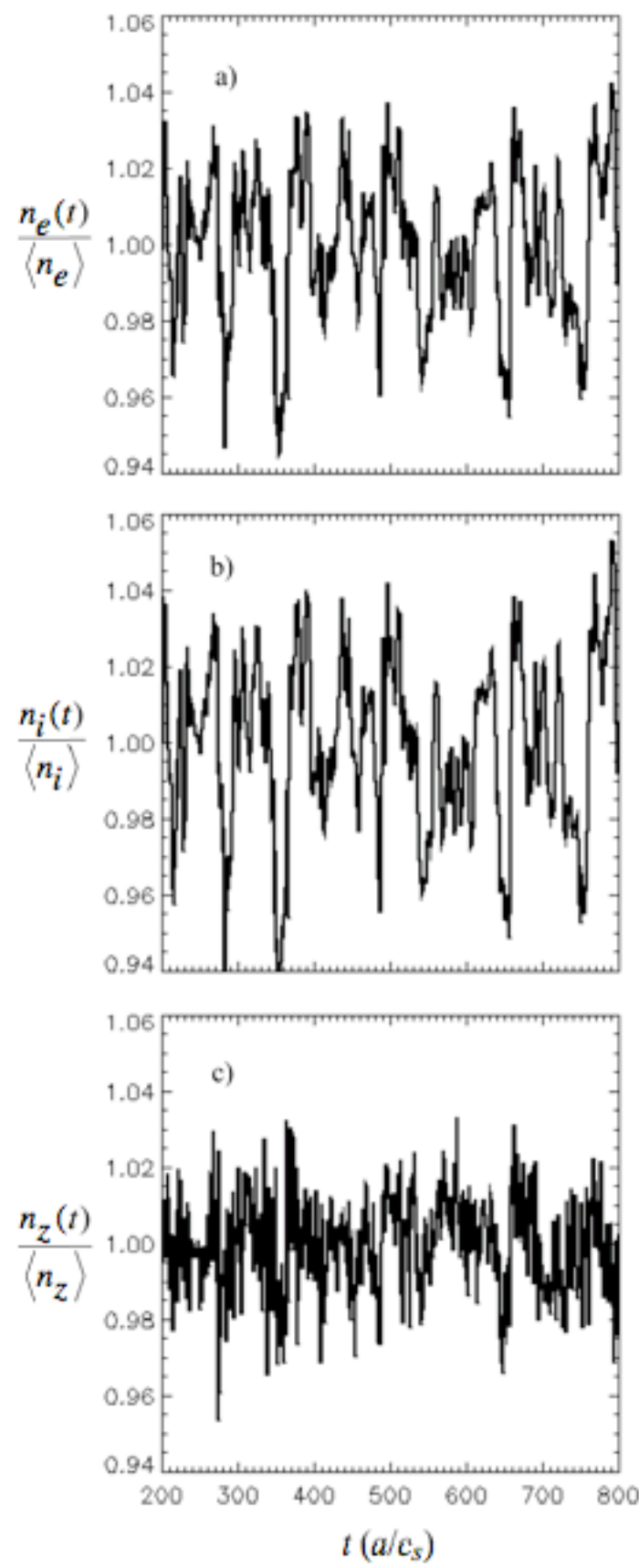

Fig. 5 

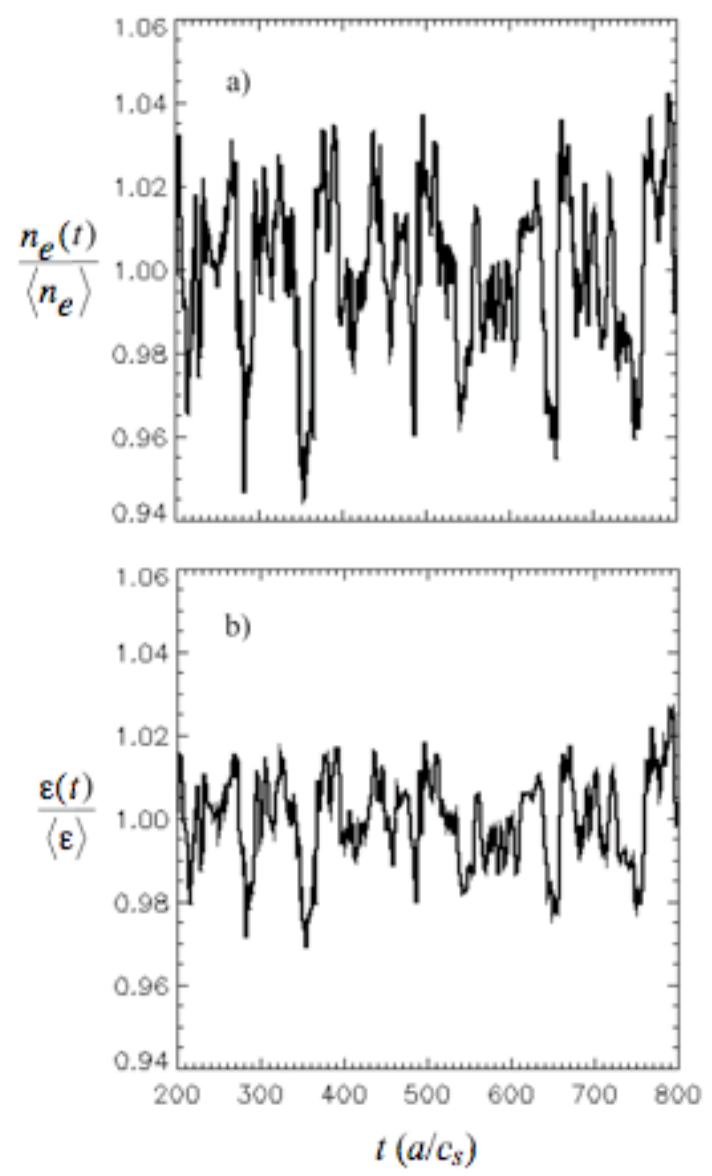

Fig. 6 


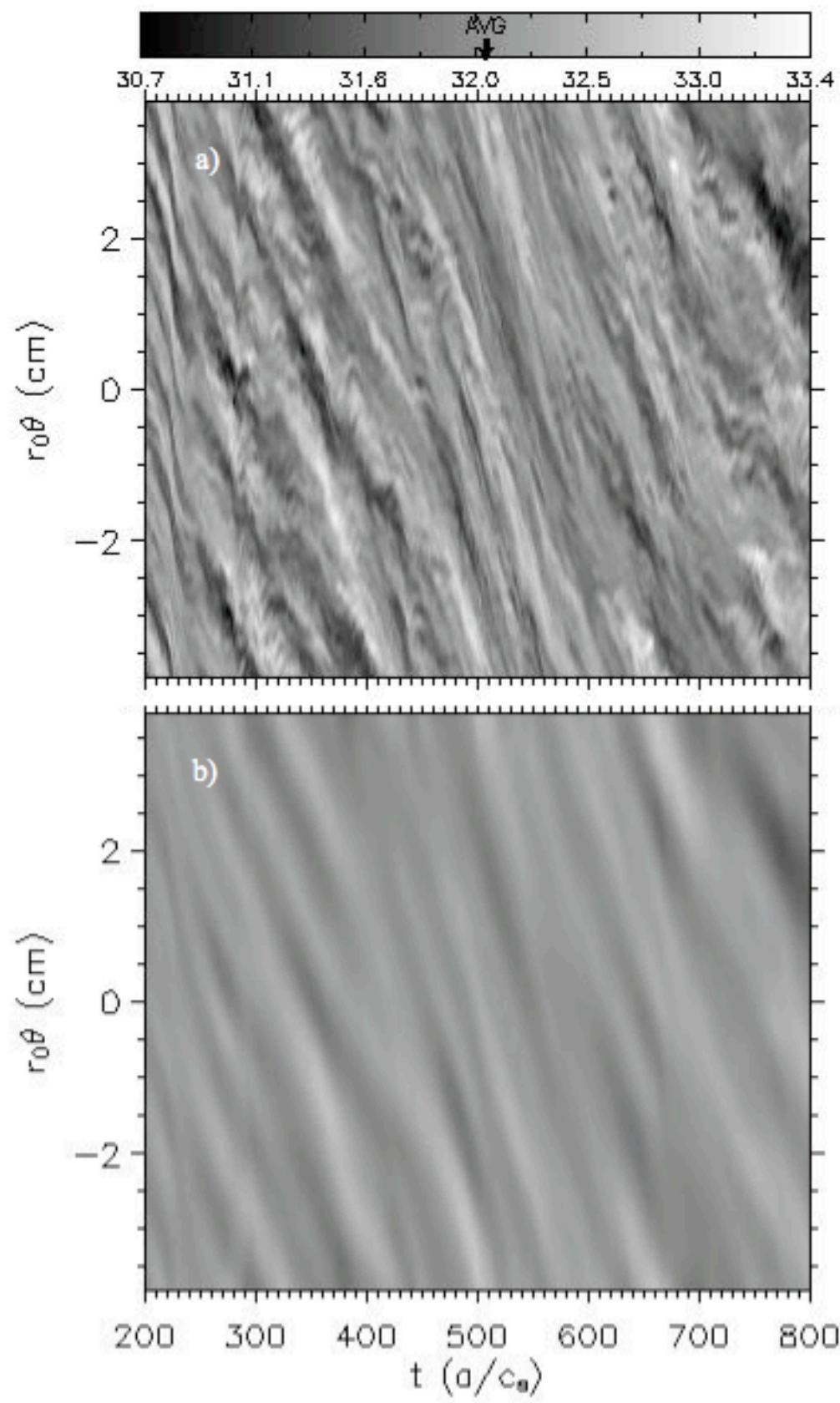

Fig. 7 

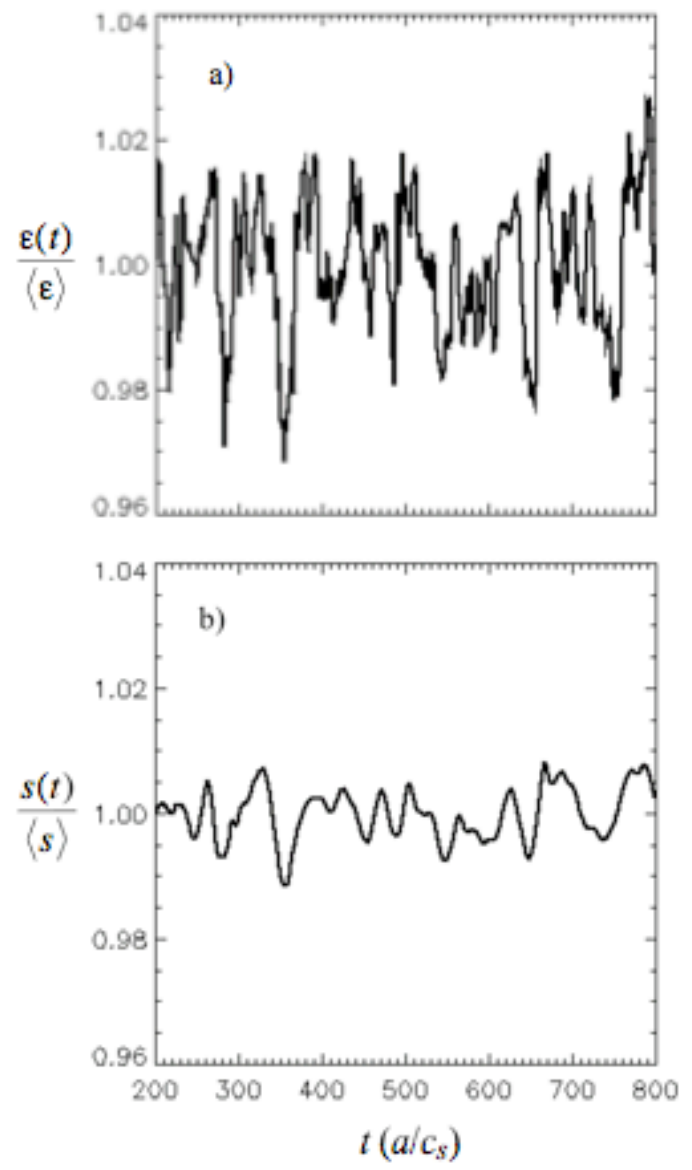

Fig. 8 


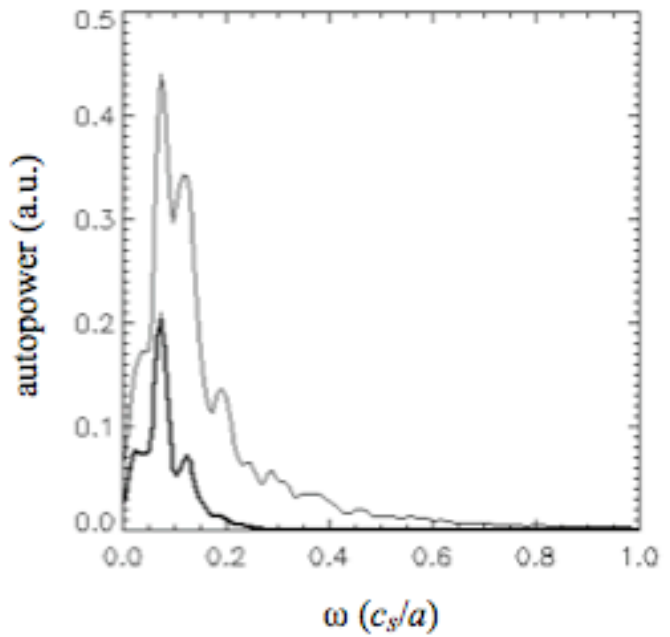

Fig. 9 